\title{
Coulisses
}

Revue de théâtre

\section{Sous un ciel muet : faust a faim. immangeable marguerite, d'Ewald Palmetshofer}

\section{Catherine Mazellier-Lajarrige}

\section{(2) OpenEdition}

12 Journals

Édition électronique

URL : https://journals.openedition.org/coulisses/207

DOI : 10.4000/coulisses. 207

ISSN : 2546-9460

Éditeur

Presses universitaires de Franche-Comté

\section{Édition imprimée}

Date de publication : 31 décembre 2011

Pagination : 23-35

ISBN : 978-2-84867-404-9

ISSN : $1150-594 X$

\section{Référence électronique}

Catherine Mazellier-Lajarrige, "Sous un ciel muet : faust a faim. immangeable marguerite, d'Ewald Palmetshofer », Coulisses [En ligne], 43 | Automne 2011, mis en ligne le 30 novembre 2016, consulté le 29 décembre 2022. URL : http://journals.openedition.org/coulisses/207 ; DOI : https://doi.org/ $10.4000 /$ coulisses.207 


\title{
Sous un ciel muet : faust a faim. immangeable marguerite, d'Ewald Palmetshofer
}

\author{
Catherine Mazellier-Lajarrige
}

1 Le Faust de Goethe ne cesse de nourrir la langue et l'imaginaire de l'espace culturel germanophone. Au théâtre, tout particulièrement dans le contexte de la "Wende» depuis l'unification allemande, de nombreuses mises en scène interrogent cette tragédie sur son actualité avec des choix dramaturgiques relevant pour la plupart d'une esthétique postdramatique. Dans les années 1990, on peut citer les mises en scène d'Einar Schleef à Francfort, de Michael Engel à Dresde, Dieter Dorn à Munich ou Christoph Marthaler à Hambourg et, plus récemment, celles de Michael Thalheimer à Berlin ou de Matthias Hartmann à Vienne ${ }^{1}$. Dans le cas d'Ewald Palmetshofer, qui avait déjà fait référence à un classique du théâtre européen pour sa première pièce hamlet ist tot. keine schwerkraft (hamlet est mort. gravité zéro ${ }^{2}$ ), le mythe de Faust a inspiré au dramaturge autrichien une variation qui transpose le texte source de Goethe dans la société contemporaine et accorde une large place au personnage de Marguerite, explicitement nommée dans le titre : faust hat hunger und verschluckt sich an einer grete (faust a faim. immangeable marguerite) ${ }^{3}$. À la structure chiastique du titre correspond son contenu anthropophage, perceptible dans un jeu de mots intraduisible sur l'homophonie entre Grete - diminutif de Marguerite - et Gräte, qui signifie "arête »: littéralement, "faust a faim et avale une grete / une arête de travers ", nous dit l'original. Le trait d'esprit et la minimisation des protagonistes par l'utilisation des minuscules - un procédé cher à l'avant-garde viennoise des années 1960-70 (Groupe de Vienne, Ernst Jandl, Oskar Pastior...) et repris chez les dramaturges autrichiennes Elfriede Jelinek et Kathrin Röggla 㡠 mettent à distance le monument littéraire que constitue le modèle goethéen. Ce titre, malgré son ironie légère, annonce le thème central du désir tout-puissant, égotique, qui engloutit l'autre et le nie en tant que sujet.

Il touche ainsi aux préoccupations majeures d'Ewald Palmetshofer, l'un des représentants majeurs de la nouvelle génération théâtrale autrichienne. Après des études de théâtre et de littérature allemande, l'auteur s'est tourné vers la psychologie, 
la philosophie et la théologie afin d'être au plus près des questions centrales à ses yeux : le mal, l'injustice, la mort - y compris et surtout dans un monde où le ciel est désormais vide, comme le dit Mani, le personnage du fils dans hamlet est mort : « Depuis que Dieu est mort, le ciel est vide, mais pas tout à fait, disons presque vide, le ciel, et c'est pourquoi on peut dire, selon une topographie générale du ciel, que certes le ciel est vide, mais qu'en tant que tel, en tant que ciel vide, le ciel est une machine $»^{4}$. Selon Mani, cette machine distribue à tous un numéro qui sera pris en compte dans le bilan général de l'humanité, à la fin des temps.

Ewald Palmetshofer commence par écrire des nouvelles dans un style très attentif au rythme, aux effets de répétitions et à la construction d'une oralité, découvre alors le plaisir performatif de les lire à haute voix, ce qui le conduit insensiblement vers l'écriture dramatique ${ }^{5}$. Après la création de hamlet ist tot. keine schwerkraft au Schauspielhaus de Vienne, le théâtre accueille également sa pièce wohnen. unter glas [habiter. sous verre] dans la mise en scène de Barbara Weber en février 2008 et propose à l'auteur une résidence pour la saison 2008-2009, qui s'achève par la création de faust hat hunger $[. . .]^{6}$. Alors que pour hamlet est mort, les allusions à la pièce de Shakespeare s'étaient immiscées dans le texte de Palmetshofer au fil de l'écriture ${ }^{7}$, le mythe de Faust élaboré par Goethe constitue véritablement le point de départ de faust a faim [...] et les références à l'hypotexte goethéen y sont légion. D'emblée, l'auteur décide d'envisager ce qu'il reste du mythe de Faust à l'époque contemporaine, « sous un ciel vide et aussi, de [son] point de vue, devant un enfer vide $»^{8}$. Dans cette perspective, Faust incarne le désir de bonheur terriblement individualiste qui caractérise nos sociétés industrialisées, peu soucieuses de poser comme exigence fondamentale la recherche d'un bonheur partagé par tous. La référence à Goethe structure cette réécriture qui s'articule, jusque dans le traitement de la langue, autour de l'absence et des implications politiques de la quête du bonheur.

\section{Réminiscences goethéennes}

L'hypotexte du plus grand classique de la littérature germanophone est présent sous diverses formes dans la réactualisation qu'en propose Ewald Palmetshofer, depuis la structure même de la pièce, composée elle aussi de 25 scènes, numérotées de -1 à 23 , jusqu'aux allusions les plus ténues. Les trois textes d'ouverture, dont Goethe a fait précéder son Premier Faust à partir de 1797-98, se retrouvent également, de manière plus ou moins visible, dans faust a faim. À la « Dédicace », qui inscrit la tragédie dans la matière temporelle et le regret des temps enfuis, fait écho une citation de François Jullien, extraite de son essai $D u$ «temps». Éléments d'une philosophie du vivre : le philosophe s'y inspire de la pensée chinoise pour dépasser l'opposition entre temporalité et éternité, entre passé, présent et futur et, face à l'angoisse de la mort, s'inscrire dans le moment présent afin de "penser une "insouciance" qui ne soit pas une fuite $»^{10}$. En guise de "Prologue sur le théâtre», Palmetshofer propose au spectateur un "Prologue à la télé ", numéroté "-1", qui sert de soubassement à l'ensemble ${ }^{11}$. De même que le directeur de théâtre, dans l'hypotexte goethéen, réclame " De l'action! / On vient pour le spectacle, on veut surtout voir $»^{12}$ (v. 90), Palmetshofer transporte cette ouverture dans notre " société du spectacle », dont Guy Debord a bien décrit les dérives et les formes d'aliénation ${ }^{13}$. Le rôle du personnage comique (lustige Person) ou bouffon, défenseur du divertissement dans le «Prologue sur le théâtre », y 
est endossé par un couple habitant le village d'enfance de la protagoniste Grete: friands de commérages, ces voisins sont la réduplication grotesque de la lustige Person. La tragédie, suite de flashbacks et de reconstitutions, s'inscrit en effet dans un cadre métadramatique, celui d'une émission de téléréalité ou d'un documentaire policier sur un cas d'infanticide, propre à satisfaire la curiosité malsaine des spectateurs. Un troisième cadre, métaphysique, intitulé "Prologue [pas de ciel] » et numéroté «0 0 " oppose d'emblée au «Prologue au ciel» la radicalité d'un monde sans Dieu, où les humains disent leur chute à travers le néant, leurs ailes "réduites en cendres et

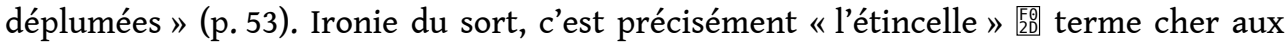
mystiques chrétiens - qui a embrasé les ailes et contraint ces anges déchus à vivre sur terre, dans le froid et l'obscurité.

5 La scène numérotée « 1 » se situe donc quelque part sur terre, plus exactement dans un appartement où se retrouvent deux couples de trentenaires petits-bourgeois - Inès et Paul, Tania et Robert -, habitués à s'inviter mutuellement pour des soirées « bring what you eat " autour d'un barbecue. Ce soir-là, ils ont décidé d'intégrer à leur petit cercle un troisième couple, Anne et Fritz, dont la vie sociale a été réduite par la naissance de leur enfant, Ismaël. Tandis que Paul s'essaie à un ridicule discours de bienvenue pour faciliter leur intégration, ou leur ingestion, deux célibataires viennent agrandir le cercle, variation moderne du cercle magique qui protège les rituels ésotériques d'influences néfastes ${ }^{14}$ : Grete, une amie d'Anne, assistante sociale tout comme elle, et Henri, un ami de Paul engagé « dans l'humanitaire ». C'est leur rencontre que racontent alternativement les trois couples tout au long des 25 scènes, depuis les premiers regards échangés jusqu'à la catastrophe inspirée de la tragédie de Marguerite, en passant par la découverte émue de goûts et d'intérêts communs. La perspective d'ensemble est donc narrative, elle fait alterner, avec un système typographique de mises en retrait correspondant à des temporalités et des situations d'énonciation différentes, des dialogues qui rejouent le passé et des commentaires après-coup, témoignages destinés aux reporters de la télévision, mettant ainsi bout à bout ces «fragments d'un discours amoureux» dont Roland Barthes a mis à nu l'immuable structure $^{15}$.

\section{Une écriture de l'absence}

6 Les prénoms des deux protagonistes, Henri et Grete, sont absents de la liste des personnages, et ce ne sont d'ailleurs que des «noms de code", voulus par le mythe. Leurs rôles doivent être distribués entre les autres personnages : "Quand le nom d'un personnage est barré d'un trait, cela signifie que le personnage en question est en train de jouer Henri ou Marguerite. On ne connaît pas leur véritable nom.» (p. 39). Dans la mise en scène de Felicitas Brucker à Vienne, l'un des trois personnages féminins qui incarnent « Grete » à tour de rôle enfilait une jupe portant l'inscription «GRETE » pour indiquer cette identité surajoutée, tandis que le personnage de Fritz, habité par le rôle de "Henri», revêtait un tee-shirt marqué à son nom ${ }^{16}$. À aucun moment les protagonistes ne sont nommés par les autres personnages : ils sont évoqués par le biais des pronoms «LUI» et «ELLE». Le nom barré est le moyen graphique qu'Ewald Palmetshofer, lecteur assidu de Lacan, a trouvé pour matérialiser l'absence de sujets littéralement «barrés ${ }^{17}$, tout en problématisant le triangle acteur / personnage / rôle et la non-identification de l'acteur au personnage. L'auteur ne voulait pas d'une Grete 
qui meure sur scène et vienne saluer à la fin, il lui fallait rendre palpable cette mort comme un manque inéluctable et irrémédiable. C'est pourquoi « Grete » est absente dès le début de la pièce ${ }^{18}$.

Quant à Henri, rejetant le "bonheur en série » (p. 75) que lui propose la société de consommation, il cherche à donner un sens à son existence à travers son engagement humanitaire dans des zones de conflit. Lorsqu'a lieu la rencontre avec Grete, il est assis derrière son ordinateur portable, en train de passer de la musique pour animer cette soirée un peu ringarde où les deux célibataires ne se sentent pas à leur place. Avant même son arrivée, Henri est annoncé comme celui que l'on attend, « le monsieur avec la musique » («den Herrn mit der Musik », p. 62), l'allemand reprenant le terme de Herr monsieur, seigneur ou encore Seigneur Dieu - qui désigne l'interlocuteur divin de Méphistophélès dans le « Prologue au ciel ». L'ordinateur, trace contemporaine de cette "mélancolie du savoir» caractéristique de Faust ${ }^{19}$, fonctionne ici comme un signe extérieur de savoir postmoderne, à l'instar de la toge pour le savant de la Renaissance.

Heureuse coïncidence, le morceau qu'Henri passe sur son ordinateur est justement la chanson préférée de Grete, mais aujourd'hui, Marguerite ne chante plus au rouet son cœur lourd et la paix enfuie. Elle sait qu'à la différence de l'héroïne de Goethe, elle n'a plus seize ans depuis longtemps et que, la quarantaine approchant, l'heure de la dernière chance est peut-être arrivée. Mais en rentrant seule chez elle, après avoir fui cette soirée ennuyeuse, elle peut reprendre à son compte la réplique que prononce la Marguerite de Goethe : «Je ne suis ni demoiselle ni belle / Et rentrerai bien toute seule à la maison $»^{20}$ (v. 2607 s.). À l'ère de la postmodernité, ce sont deux êtres désabusés qui se croisent. La femme d'âge mûr est sans complaisance à l'égard de son comportement de gamine énamourée, elle s'en veut d'être dupe de cette attirance qui voudrait lui faire oublier que son corps de provinciale «trahit sans cesse sa provenance» (p. 111), tout comme Henri, dans un monologue qui fait écho à la scène « Nuit » chez Goethe ${ }^{21}$, raille sa propre incapacité à retenir le bonheur dans son for intérieur aux parois lisses, aussi lisses que le poêle de faïence dans le cabinet d'étude de Faust.

Que devient alors l'esprit diabolique qui, chez Goethe, sort de derrière le poêle pour apparaître à Faust sous l'apparence d'un étudiant ambulant? Métamorphose contemporaine, il vient se loger dans l'esprit de négation qui anime Henri et Grete : ce sont deux êtres "qui toujours nient $»^{22}$, trouvent dans ce principe de négation un dénominateur commun et croient "que dire " non " ensemble / ça revient à un oui " (p.143), sans voir que l'addition de deux souffrances ne peut avoir pour somme le bonheur tant désiré. Le rôle de Méphisto est également endossé par le groupe qui gravite autour du couple : selon l'auteur, le groupe « séduit, dirige le désir de Henri, le conduit vers un objet. Il a le côté entremetteur de Marthe et de Méphisto et tente de persuader Henri de rester auprès de cet objet. $»^{23}$

10 Méphisto n'est ici l'adversaire d'aucun Dieu, et l'on peut observer que les différentes notions et valeurs métaphysiques autour desquelles s'articule la pièce de Goethe sont déplacées ou perverties. Le Dieu judéo-chrétien ne subsiste plus qu'en filigrane, dans le prénom de l'enfant d'Anne et Fritz: Ismaël, qui signifie en hébreu «le Seigneur Dieu entend", permet une remotivation ironique de l'expression idiomatique "das Kind beim Namen nennen », utilisée en sa présence 㡠 littéralement : appeler l'enfant par son nom医, autrement dit "appeler les choses par leur nom ${ }^{24}$. Or, le Dieu de l'Ancien Testament, qu'Ismaël contient dans son nom, est précisément celui qui ne peut être nommé et qui, selon Palmetshofer, est aujourd'hui le grand absent ou du moins reste 
sourd au tragique de la condition humaine. C'est peut-être l'une des raisons pour lesquelles, jusqu'à la fin de la pièce, l'identité de l'enfant retrouvé mort dans la forêt, aux côtés de Grete, reste indéterminée. Jusqu'au bout, le doute plane : il pourrait s'agir d'Ismaël, l'enfant-Dieu, confié par ses parents à Grete le temps d'un week-end entre amis.

Dans ces conditions, une alliance de Faust avec Méphisto ne peut se dessiner qu'en creux. De même que Goethe ne pouvait plus reprendre la forme traditionnelle du pacte avec le diable, ancrée dans la foi chrétienne et une grande peur du diable, et qu'il dut attendre trente ans avant de trouver une forme qui fût adaptée à son époque déjà marquée par la sécularisation - celle du pari dans la scène "Cabinet d'étude II » -, Ewald Palmetshofer ne peut reprendre tel quel cet élément dans son faust postmoderne. Aujourd'hui, déclare Fritz, "peut-être qu'il faut parier sur l'existence d'une substance dans l'être humain » (p. 139). Le pari est dans la relation à l'autre, et il subsiste à l'état de trace lexicale, vidée de son contenu sémantique premier, dans la dernière scène de la pièce, où il équivaut à un pronostic négatif sur l'avenir du couple Elle-Lui, à propos duquel Tania déclare : «je ne parlerais pas de pari» (p. 193). De la même façon, la terrible damnation qui attendait le Faust de la Renaissance n'est plus chez Palmetshofer qu'un lointain souvenir, figé dans un juron répété en toute circonstance : «verdammt ", rendu dans la traduction française par «bon dieu ».

Cette dilution sémantique est relayée par l'ellipse de termes attendus, comme celui de «religion» dans une parodie de la célèbre Gretchenfrage. Dans sa réponse à cette question de Margarete, qui presse Henri de s'exprimer sur sa foi - dans l'original: "Nun sag', wie hast du's mit der Religion?» (v. 3415) -, le personnage de Goethe déplace la question de la transcendance vers l'immanence pour proclamer, sur fond de panthéisme, une religion du sentiment sacralisé, élevé au rang de valeur suprême : «Et si par ce sentiment tu es heureuse, / Nomme-le comme tu voudras, / Bonheur ! Cœur ! Amour! Dieu! / Moi, je n'ai aucun nom / Pour cela! Le sentiment est tout, / Le nom n'est que bruit et fumée / Qui nous voile l'éclat des cieux.» (v. 3452-3458). Chez Palmetshofer aussi, dans une scène littéralement centrale, puisqu'il s'agit de la treizième sur un ensemble de 25 scènes, la réponse à la question cruciale posée par Grete contient un déplacement de la "religion» vers "l'amour", mais ce dernier terme est trop lourd, trop difficile à prononcer, prisonnier de la polarité entre matière et idéal. Il reste inéluctablement en creux, perceptible entre les bribes d'une profession de foi fragmentaire et disloquée :

comment voit-il, et lui il répond, il faudrait déjà commencer par soi-même, oui, il dit, et cet, oui, pour soi-même, tu sais, c'est cela qui fait naître un, oui, pour l'autre, intérieur, complètement différent de cette enveloppe extérieure qu'il appelle le corps, si soi-même on, oui (p. 131)

Le terme omis dans ce fragment, c'est le mot « amour », celui qui viendrait combler les manques mais n'y parvient jamais, car pour cela, il faudrait déjà commencer par s'aimer soi-même, de sorte que le « oui » prononcé comme un tic de langage ne peut équilibrer un «non » fondamental.

14 Si l'amour de soi et de l'Autre est si problématique, le bonheur est tout aussi fragile. «Le bonheur, c'est un chevreuil », déclare Tania à la scène 7 (p. 109), choisissant cette image insolite, évocatrice de Bambi. Ce symbole de l'extrême vulnérabilité est lié à la fugacité de l'instant présent, soulignée par des bribes du célèbre vers de Goethe sur 
l'objet du pari faustien, « Verweile doch du bist so schön » (v. 1700 : « Attarde-toi, tu es si beau!»):

PAUL attarde-toi

TANIA mon chevreuil

INÈS si beau (p. 185)

Retenir l'instant fugace est le désir d'une humanité qui cherche dans l'immanence une parcelle de divin. Chez Goethe, Faust cherche à connaitre, au moyen de la magie, « ce par quoi le monde / Est, en son tréfonds, maintenu ensemble » (v. 382-383), tandis que chez Palmetshofer, Henri se met en quête du tréfonds de l'être humain, de ce que l'on pourrait se risquer à nommer une " âme » : la " petite étincelle de lumière " chère aux mystiques. Mais devant ses interrogations, le "ciel étoilé ", réminiscence kantienne, reste muet: «dans le doute tu lèves la tête vers le haut, regard vertical cherche, en haut, nuque figée, une étincelle de lumière, le ciel étoilé se tait, tu cherches quand même, tu n'appelles plus ça le bonheur maintenant mais la vérité [...]» (p. 77). Et des âmes, il ne subsiste plus que « des mèches de bougies carbonisées / froides » (p. 57).

La nostalgie d'une substance s'exprime à travers le terme «Kern" (littéralement: noyau), récurrent dans cette pièce et d'une manière générale chez Palmetshofer, notamment dans hamlet est mort et dans tier. man wird doch bitte unterschicht [animal. on glisse tout de même vers les couches inférieures ${ }^{25}$. Ce terme fait écho à la réplique, devenue proverbiale, du Faust de Goethe, lorsque le caniche qui l'a suivi dans son cabinet d'étude se métamorphose en "étudiant voyageur »: «Das war also des Pudels Kern!» (v. 1323 : «C'était donc le secret du barbet!»). Comme le Faust de Nikolaus Lenau, en 1836, dissèque des corps à la recherche du secret de l'humain - image forte qui a inspiré Mark Ravenhill pour son Faust is Dead $[1996]^{26}$, son avatar chez Palmetshofer fouille de manière obsessionnelle le corps de Grete à la recherche d'une substance, d'un « noyau " qui reste introuvable ${ }^{27}$. La femme abandonnée exprime son désespoir dans un monologue qui fait écho à la scène "Cathédrale » du Faust I, où le mauvais esprit vient tourmenter Marguerite et brandir le courroux céleste du dies irae. Elle en tire la conséquence la plus radicale qui soit par le meurtre de l'enfant, de cette matière née entre deux corps d'une idée de l'amour.

L'évidement des valeurs ontologiques et métaphysiques se traduit par une écriture de l'absence, de l'ellipse et du fragment, dont nous avons donné un aperçu. Un procédé cher à l'auteur - qui n'utilise pourtant jamais les points de suspension - consiste à laisser en suspens la fin des phrases, et ce non pas en signe d'une impossible communication entre les êtres, mais parce qu'il leur est nécessaire de reprendre leurs formulations, encore et toujours, dans une vaine recherche de l'adéquation entre le mot et à la chose. La langue porte la marque de l'inadéquation permanente entre ce qui est dit et la vérité de l'être, symptôme d'une éternelle insatisfaction. Ainsi que l'a déclaré Ewald Palmetshofer au critique Andreas Klaeui, des éléments de vérité ne peuvent devenir perceptibles que dans la rupture, «ex negativo et non dans la mise en scène d'un sens affirmatif $»^{28}$.

18 C'est la musicalité de la langue, son rythme, qui sont signifiants, indépendamment du contenu sémantique, pour ces personnages en quête d'une langue. Le rythme naît aussi du télescopage entre présent et passé, dialogues commentant la catastrophe et reconstitution des actions qui l'ont précédée, parties dialogiques et blocs narratifs monolithiques, un procédé choral qu'Ewald Palmetshofer a poussé jusqu'au raffinement extrême dans sa dernière pièce, tier. man wird doch bitte unterschicht. Cette 
tension entre des temporalités et des identités différentes, source d'un effet de distanciation permanent, s'exprime également dans les scènes où les couples rejouent les personnages de Grete et Henri, à la manière d'une pièce dans la pièce. Anne, Robert, Inès deviennent les acteurs d'une illusion théâtrale et démultiplient la distanciation en jouant la rencontre amoureuse :

ANNE alors, toi aussi tu t'es cassé, elle dit

ROBERT oui enfin pas aussi vite que toi

INÈS et ils se mettent à rire

sûr qu'ils se mettent à rire

ANNE je connaissais personne et Anne

elle comprendra

INÈS elle a de ces copines, Anne,

des copines comme moi, qui foutent le camp

ANNE pas vraiment la fibre sociale, cette copine d'Anne, moi donc

pas la fibre sociale en privé

mais ils comprendront, sûrement, et Anne aussi

puisque je connaissais personne (p. 115)

Le sujet - «moi donc» 原 se dissout dans les multiples jeux de miroir. Dans ces conditions, comment penser l'être dans le monde?

\section{De la métaphysique à la politique de la relation}

Le questionnement métaphysique, la pensée de la mort et la quête du bonheur, face à cette donnée inéluctable sont indissociables, chez Palmetshofer, d'une réflexion politique sur la place de l'homme dans la société et les lois impitoyables qui régissent les rapports humains. En particulier, le mode de relation entre les êtres est décrit comme relevant de la pure économie capitaliste ; selon Henri, l'homme spécule sur une intériorité chez ses congénères - avatar postmoderne de l'âme -, créant ainsi une valeur ajoutée :

des milliers de fois déjà, tu as déjà investi dans un être humain pour une production de plus-value, tu as spéculé sur une intériorité qui lui insuffle une substance et produise en contrepartie une création de valeur, peut-être, dont on n'aurait pas cru capable cette surface, cette surface d'être humain et qui laisserait transparaître une substance à contre-jour, qui devrait la laisser transparaitre, à condition qu'on y injecte et qu'on y transfère un capital suffisant, et que, par un effet secondaire de ta spéculation, une plus-value d'intériorité soit fabriquée dans cette surface d'être humain et que le capital t'ait appris à créer ex nihilo, et tu te projettes vers une création, une création de valeur humaine et tu abordes cet être humain avec un endettement que tu peux à peine te permettre, et tu lui as déjà financé son intériorité des milliers de fois [...] (p. 139)

21 Cette vision économiste est corroborée par la possibilité d'une lecture sociale de la relation entre Henri et Grete, au sens d'un antagonisme entre bourgeoisie et prolétariat, entre grande ville et province, centre et périphérie. Grete l'exprime dans une attaque qui cristallise ainsi leurs oppositions :

seulement que le prolétariat auquel j'appartiens ne tend qu'à l'embourgeoisement et que la bourgeoisie comme lui continue de tendre vers l'idéal

et que moi je suis pas née pour l'Essentiel (p. 171)

L'antagonisme est sensible à travers une certaine vision de la nature, idéalisée dans la bourgeoisie urbaine, tandis que Grete, par l'horreur de son acte, y oppose le réalisme d'une violence primitive en réaction à une violence relationnelle qui ne fait 
qu'exacerber son sentiment d'être déclassée. La scène de la découverte des corps dans la forêt est d'autant plus forte à cet égard qu'elle met la terrible réalité à distance par une parodie, à la manière d'Elfriede Jelinek ${ }^{29}$, de discours visant à idéaliser la nature, notamment la belle forêt autrichienne :

ROBERT la nature est restée vierge ici

INÈS la femme spécialiste de la cueillette et de la chasse

TANIA on lâche les chiens

PAUL le médecin secouriste bondit à travers les fourrés

INÈS la civière, vite, bon dieu

PAUL l'hélicoptère de secours ne peut pas atterrir ici dans la forêt

ROBERT il atterrira sur le terrain de foot

INÈS une photo qu'on a malheureusement laissé passer

TANIA puis les brancardiers qui slaloment entre les sapins avec leur civière

INÈS leur couleur rouge dans la forêt mixte un enchantement

rouge vert, superbe contraste

ROBERT l'hélicoptère atterrit pile dans le cercle au milieu du terrain de foot

balaie la marque blanche de la chaux

PAUL l'équipe pleure

TANIA un chevreuil interrompt son chant (p. 157)

Si l'antagonisme et la lutte sont au cœur des rapports entre les humains, se pose alors la question du fonctionnement du corps social. Soucieux d'unité et de totalité, ce dernier cherche à intégrer, assimiler l'autre, le nouveau, l'étranger, tel Paul, avec son discours de bienvenue destiné à intégrer dans le groupe de quatre amis déjà constitué le couple de "nouveaux», Anne et Fritz, restés à l'écart de la société depuis la naissance de leur enfant Ismaël. Ou encore à la manière anthropophage de Faust, avalant une Marguerite de travers. Elle fait écho à l'ingestion du corps christique à travers l'eucharistie, dont l'image sous-tend cette réplique de Robert :

ROBERT et la voix du peuple ne s'y trompe pas, les gens se rassemblent en parlant et en bouffant et ouvrent pleins de grâce la voix du peuple nous la faisons entendre et invitons, invitons les autres dans la gueule du peuple par l'ouverture du corps collectif, corps gras, où il reste un peu de place pour quelques autres, qu'on intègre et incorpore volontiers (p. 93)

Le "Streben ", cette aspiration à la connaissance et à la vérité qui justifie Faust in fine et le sauve dans la tragédie de Goethe ${ }^{30}$, est réduit ici à un désir d'assimiler autrui au sens propre, et l'acte amoureux n'est plus qu'une ingestion de l'autre, dans une quête désespérée de la complémentarité et de la totalité perdue, de sorte que le mythe de l'homme-sphère, développé dans Le Banquet de Platon, est encore bien vivace ${ }^{31}$. L'être humain consomme ses congénères, chaque cadavre vient engraisser un peu plus le Capital, déclare Palmetshofer ${ }^{32}$. Même les projets humanitaires, comme ceux qui procurent à Henri une bonne conscience, ne relèvent pas d'une véritable politique pour les autres ${ }^{33}$. Son interventionnisme humanitaire transpose au début du xxI ${ }^{e}$ siècle l'activité colonisatrice que décrit la fin du Second Faust, celle-là-même qui a permis d'interpréter le protagoniste goethéen comme une figuration des dangers de la modernités ${ }^{34}$.

Clin d'œil à ce projet titanesque, où Faust se pose en bienfaiteur de l'humanité par la réalisation d'une grande digue - balayée par une prédiction de Méphisto -, Henri, lui, contient les flots en creusant des latrines dans des zones de conflit mais reste sourd à la catastrophe qui se prépare à côté de lui. Grete, de son côté, regarde " le purin » couler le long de ses jambes dans la forêt où elle commet l'irréparable ${ }^{35}$. Les références aux excréments sont nombreuses, complétées par l'image du poison. Mais tandis que le 
poison reste extérieur à l'homme chez Goethe - sous la forme de la fiole pour Faust tenté par le suicide, ou encore du « somnifère » qui sera fatal à la mère de Marguerite -, c'est le corps humain lui-même qui est, à présent, déclaré toxique :

le poison sécrété par les cadavres, ça n'existe pas

c'est un mythe

ça n'existe pas, ce poison

c'est seulement quand le corps est vivant que c'est un poison (p. 121)

Alors, où trouver l'absolu ardemment désiré ? Nulle alchimie ne vient transmuter la vile matière en or : Henri n'a pas trouvé ce « tout » auquel il aspire. Aucune conciliation ni réconciliation n'est possible. Cependant, l'absolu vient se loger précisément dans la radicalité de Grete, personnage plus faustien que Faust lui-même, selon Ewald Palmetshofer ${ }^{36}$. L'identité indéterminée de l'enfant mort, à l'image de l'indétermination de la valeur " être humain ", permet de transposer le tragique du destin de Grete, ainsi arraché à l'évidence figée du mythe, pour retrouver l'horreur première.

La Grete d'Ewald Palmetshofer «n'est pas la première »" ${ }^{37}$, mais le dramaturge autrichien parvient pourtant à faire ressentir son unicité et la douloureuse radicalité de son absence. Par le moyen des personnages "barrés", il rend palpable le manque irrémédiable que constitue la mort d'un être. Et si cette transposition contemporaine du mythe de Faust ne peut laisser indifférent, c'est qu'elle interroge avec une acuité particulière la capacité de l'homme à concevoir un bonheur qui ne soit plus individualiste mais tourné vers l'Autre, attentif aux liens entre les êtres, à défaut d'être relié à une transcendance. Ewald Palmetshofer illustre ainsi, dans un gestus cher à son mentor Alain Badiou, à quel point le religieux a trouvé refuge dans le politique. Et le recours au mythe lui permet précisément de poser la question de la singularité : celle $\mathrm{du}$ « bel instant » et celle de la catastrophe.

\section{NOTES}

1. Cf. Im Auftrieb. Grenzüberschreitungen mit Goethes «Faust» in Inszenierungen der neunziger Jahre, hg. v. Hans-Peter Bayerdörfer, Tübingen : Niemeyer, 2002. Le travail du metteur en scène Michael Thalheimer au Deutsches Theater entre 2004 et 2006 a suscité un colloque et une publication, "Verweile doch» - Goethe Faust heute, hg. v. Michael Jaeger, Roland Koberg, Bernd Stegemann, Henrike Thomsen, Berlin, Henschel 2006.

2. Cette pièce, créée en novembre 2007 au Schauspielhaus de Vienne (mise en scène : Felicitas Brucker) et traduite en français par Laurent Muhleisen, a donné lieu à une création radiophonique sur France Culture le 17 avril 2010 (réalisation : Alexandre Plank).

3. La pièce sera citée dans l'édition bilingue : Ewald Palmetshofer, faust hat hunger und verschluckt sich an einer grete / faust a faim. immangeable marguerite, trad. Laurent Muhleisen, Catherine Mazellier et Hilda Inderwildi, Toulouse, Presses Universitaires du Mirail, « Nouvelles Scènes Allemand », 2011, en abrégé : faust hat hunger, suivi du numéro de page. En accord avec l'auteur, 
l'usage des minuscules pour « faust » et « grete » ne sera conservé que dans le titre de la pièce. La polysémie de « marguerite» dans le titre français - héroïne goethéenne et fleur qu'effeuillent les amoureux 员 permet de transposer le jeu de mots sur Grete / Gräte dans l'original.

4. Cf. Ewald Palmetshofer, hamlet est mort. gravité zéro, trad. Laurent Muhleisen, tapuscrit non publié. L'Arche est agent de l'auteur en France. E. Palmetshofer, hamlet ist tot. keine schwerkraft, in Theater Theater. Aktuelle Stücke 18, Frankfurt a.M. : Fischer, 2008, p. 451 : «Seit Gott tot, ist der Himmel leer, aber nicht ganz, also fast leer, der Himmel, und drum kann man in einer allgemeinen Topographie des Himmels sagen, dass der Himmel selber zwar leer, aber als solcher, als leerer Himmel ist der Himmel eine Maschine ».

5. $C f$. le portrait de l'auteur par Andreas Klaeui, «Der radikale Theatertheologe» sur le site nachtkritik.de :

http://www.nachtkritik-stuecke08.de/stueckdossier3/portraet-ewald-palmetshofer.

6. Création le 2 avril 2009 au Schauspielhaus de Vienne (mise en scène : Felicitas Brucker).

7. Cf. à ce sujet les sept questions posées à Ewald Palmetshofer sur le site nachtkritik.de, «Sieben Fragen an Ewald Palmetshofer » :

http://www.nachtkritik-stuecke2010.de/ewaldpalmetshofer/sieben-fragen.

8. Ibid. : « die Frage, was bleibt von Faust unter den Vorzeichen der Gegenwart und unter einem leeren Himmel und von mir aus auch vor einer leeren Hölle».

9. François Jullien, Du «temps ». Éléments d'une philosophie du vivre, Paris, Grasset, 2001.

10. faust hat hunger, p. 39.

11. La pièce est également rythmée par un «Interlude à la télé » (scène 15) et s'achève sur un «Épilogue devant la télé » (scène 23).

12. La tragédie de Goethe sera citée dans la nouvelle traduction : Goethe, Faust, édition établie par Jean Lacoste et Jacques Le Rider, Paris, Bartillat. Nous indiquerons simplement le numéro de vers.

13. Guy Debord, La Société du Spectacle, Oaris, Buchet-Chastel, 1967.

14. Alors qu'Henri manque encore à l'appel, Paul constate l'incomplétude du groupe : «en tout cas nous sommes donc sept aujourd'hui / enfin, pas tout à fait encore, / donc, presque sept » (faust hat hunger, p.63). L'illusion de la totalité symbolisée par le chiffre 7 est révélée par l'intrusion d'un huitième personnage, Grete, qui vient briser cette harmonie factice et reste surnuméraire.

15. Roland Barthes, Fragments d'un discours amoureux, Paris, Seuil, 1977.

16. Cf. l'article de Franz Wille, « Nette Nachbarn », in Theater heute 6/2009, p. 44-45.

17. Sur les philosophes qui ont marqué Palmetshofer (Lacan, Saussure, Deleuze, Badiou), cf. l'éclairante introduction de Sylvie Arlaud, «faust hat hunger : un reader's digest ? ", in : faust hat hunger, p. 9-36; en particulier p. 10 et 14 pour l'influence de Lacan.

18. Cf. l'entretien avec Ewald Palmetshofer reproduit dans le présent numéro, citation p. $22:$ : Si l'on prend la mort au sérieux, il s'agit de la faire ressentir comme un fait irrémédiable, comme le manque d'un être. On ne peut donc pas jouer à mourir, au contraire, le personnage doit être absent dès l'origine. Nous avons six personnages sur scène, et à un moment, on a le sentiment qu'il y en sept, puis huit. Mais il y a cette femme morte qui manque. " Selon Palmetshofer, il s'agissait de marquer une gradation dans la représentation de l'absence : seul Fritz joue Henri, parti en Afrique au moment de la catastrophe, tandis que trois personnages féminins se partagent le rôle de Grete, radicalement absente (information donnée dans un entretien à l'auteur de ces lignes).

19. Jean-Yves Masson (éd.), Faust ou la mélancolie du savoir, Paris, Desjonquières, «Littérature \& Idées ", 2003. L'éditeur de cet ouvrage y évoque les formes de la mélancolie chez Faust, dont celle « de ne pouvoir atteindre à la maîtrise de la totalité des savoirs comme pouvaient y prétendre les savants antiques et, encore, les premiers humanistes de la Renaissance » (p. 10).

20. Nous citons ici la traduction d'Henri Lichtenberger, Paris, Aubier Montaigne, 1976. 
21. Ce monologue, placé à la scène 2 , était initialement prévu à la première scène, afin de souligner le parallélisme avec le Faust de Goethe. Mais à l'épreuve du plateau, l'auteur a modifié l'agencement des scènes afin de rendre l'action plus compréhensible.

22. faust hat hunger, p. 143 : « nier, voilà ce qu'ils veulent ».

23. «Un personnage plus faustien que Faust », art. cit., p. 17-22.

24. faust hat hunger, p. 67 : «ensuite Inès commence avec ce putain de cantique de merde / pourquoi ne pas appeler tout de suite les choses par leur nom ".

25. La pièce tier. man wird doch bitte unterschicht est reproduite dans le cahier détachable de la revue Theater heute $10 / 2010$.

26. Cf. l'entretien avec Mark Ravenhill in Mireia Aragay, Hildegard Klein, Enric Monforte, Pilar Zozaya (éd.), British Theatre of the 1990s, Houndmills, Palgrave Macmillan, p. 96-97. La pièce de Mark Ravenhill s'appuie sur la biographie de Michel Foucault.

27. faust hat hunger, p. $171:$ : il [...] a cherché la lumière à l'intérieur de moi en me baisant comme un fanatique / a pas trouvé de grand tout à l'intérieur de moi. »

28. " Momente von Wahrheit können nur im Bruch sichtbar werden, “ in Ex-negativo-Momenten, nicht im affirmativen Sinn inszeniert. Wahrheit ist Misstrauen der Sprache gegenüber." ” (portrait de Andreas Klaeui, art. cit., cf. Note 5).

29. Cf. par ex. Elfriede Jelinek, Oh Wildnis, oh Schutz vor ihr [1985], Reinbek, Rowohlt, 2004, trad. Yasmin Hoffmann et Maryvonne Litaize, Méfions-nous de la nature sauvage, Nîmes, Jacqueline Chambon, 1995.

30. «Wer immer strebend sich bemüht, den können wir erlösen » / « Celui qui, dans son constant effort, n'épargne pas sa peine / Celui-là, nous pouvons le sauver ", déclare le chœur des anges à la fin du Second Faust, v. 11936/37.

31. Sur le mythe de l'androgyne chez Platon, évoqué par l'auteur, $c f$. Ewald Palmetshofer, « Liebe deine Katastrophe ", in Theater heute, Jahrbuch 2008, p. 76-80.

32. Ibid. : « So wird auch das Kadaver mit jedem Kadaver, den es frisst, nur feister [...]».

33. Sylvie Arlaud, art. cit., p. 33, souligne ici l'influence d'Alain Badiou, qui a montré dans son essai L'Éthique, « que les œuvres caritatives et les ONG, dans leur victimisation des êtres humains, c'est-à-dire dans leur réduction de l'homme à sa condition d'animal vivant, révélaient leur véritable visage impérialiste. "

34. Cf., entre autres, Ulrich Gaier, Fausts Modernität, Stuttgart, Reclam, 2000.

35. faust hat hunger, p. 173. Cf. aussi p. 77, où Faust déclare : « nous sommes des latrines ».

36. $C f$. l'entretien avec l'auteur dans le présent volume, art. cit.

37. Ce vers célèbre du Faust de Goethe («Sie ist die erste nicht») est cité dans faust hat hunger, p. 182.

\section{INDEX}

Palavras-chave : intertextualidade

oeuvrecitee Faust, faust hat hunger und verschluckt sich an einer grete / faust a faim. immangeable marguerite

Mots-clés : intertextualité

Keywords : intertextuality

Palabras claves : intertextualidad 
AUTEUR

CATHERINE MAZELLIER-LAJARRIGE

Université de Toulouse II-Le Mirail, CREG 